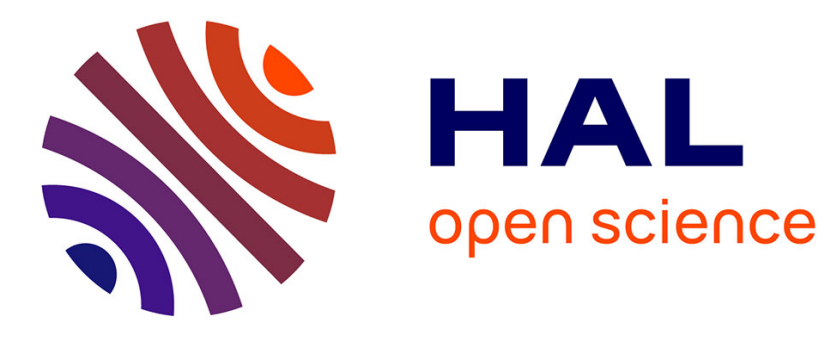

\title{
Robust RANSAC-based blood vessel segmentation
}

Ahmed Yureidini, Erwan Kerrien, Stéphane Cotin

\section{To cite this version:}

Ahmed Yureidini, Erwan Kerrien, Stéphane Cotin. Robust RANSAC-based blood vessel segmentation. SPIE Medical Imaging, Feb 2012, San Diego, CA, United States. pp.8314M, 10.1117/12.911670 . hal00642003

\section{HAL Id: hal-00642003 https://hal.inria.fr/hal-00642003}

Submitted on 12 Oct 2012

HAL is a multi-disciplinary open access archive for the deposit and dissemination of scientific research documents, whether they are published or not. The documents may come from teaching and research institutions in France or abroad, or from public or private research centers.
L'archive ouverte pluridisciplinaire $\mathbf{H A L}$, est destinée au dépôt et à la diffusion de documents scientifiques de niveau recherche, publiés ou non, émanant des établissements d'enseignement et de recherche français ou étrangers, des laboratoires publics ou privés. 


\title{
Robust RANSAC-based blood vessel segmentation
}

\author{
Ahmed Yureidini $^{a b}$, Erwan Kerrien ${ }^{a}$ and Stéphane Cotin ${ }^{b}$ \\ ${ }^{a}$ Magrit Project-team, Inria Nancy Grand-Est/LORIA, France; \\ ${ }^{b}$ Shacra Project-team, Inria Lille Nord-Europe, France.
}

\begin{abstract}
Many vascular clinical applications require a vessel segmentation process that is able to extract both the centerline and the surface of the blood vessels. However, noise and topology issues (such as kissing vessels) prevent existing algorithm from being able to easily retrieve such a complex system as the brain vasculature. We propose here a new blood vessel tracking algorithm that 1) detects the vessel centerline; 2) provides a local radius estimate; and 3) extracts a dense set of points at the blood vessel surface. This algorithm is based on a RANSAC-based robust fitting of successive cylinders along the vessel. Our method was validated against the Multiple Hypothesis Tracking (MHT) algorithm on 10 3DRA patient data of the brain vasculature. Over 744 blood vessels of various sizes were considered for each patient. Our results demonstrated a greater ability of our algorithm to track small, tortuous and touching vessels (96\% success rate), compared to MHT (65\% success rate). The computed centerline precision was below 1 voxel when compared to MHT. Moreover, our results were obtained with the same set of parameters for all patients and all blood vessels, except for the seed point for each vessel, also necessary for MHT. The proposed algorithm is thereafter able to extract the full intracranial vasculature with little user interaction.
\end{abstract}

\section{DESCRIPTION OF PURPOSE}

The segmentation of vascular structures is particularly valuable for diagnosis assistance, treatment and surgery planning. A wide range of applications may benefit from an improved blood vessel segmentation process: quantitative studies of pathologies (e.g. stenoses), automated vascular navigation, accurate blood flow computation and computer-based simulations for surgeons education. In such cases, a mere surface depiction is not enough and the extraction of the vessel medial axes is also required.

Vascular segmentation has resulted in a vast literature. ${ }^{1}$ Many previous works extract the vessel centerline tree, and claim to be robust to the kissing vessel issue: ${ }^{2-4}$ two vessels may happen to be locally tangent, or a vessel may run along a dense structure, e.g. an aneurism or bone. Such works address this issue by using a shape prior. But the downside of this assumption is that the vessel cross-section is circular, which is not always true, especially for large vessels. Thus, a trade-off is usually made between accuracy and robustness. Our work aims at preserving this desired robustness against noise and topology issues while not compromising accuracy on extraction of the vessel surface and its centerline.

This paper presents a tracking procedure which builds a vessel tree through successive robust fitting of cylinders to the image. Thereby, our dedicated tracking process: delineates the centerline of the vessel, supplies a local estimation of its radius and robustly extracts dense points on the vessel surface.

The reminder of this paper is organized as follows. Our RANSAC-Based Tracking (RBT) algorithm is introduced in Section 2.1. In Section 2.2, we describe the methodology of comparison between our proposal and the Multiple Hypothesis Tracking (MHT) algorithm. Section 3 discusses the results produced by both procedures on a data set of 10 patients, and conclusions are presented in Section 4.

\section{METHOD}

\subsection{Vessel tracking algorithm}

Efficient solutions for tracking blood vessels rely on the prior assumption that the shape is locally a cylinder. ${ }^{2,4}$ Nevertheless, previous works have hard times dealing with a large range of vessel diameters. ${ }^{4}$ Moreover, the prior is often imposed too strongly, leading to vessels with circular cross-sections. The algorithm introduced in this section suggests rather than imposes a cylinder shape as a guide to select points, in a robust manner, on the local surface of the blood vessels. See also Figure 1 for a depiction of the main steps. 


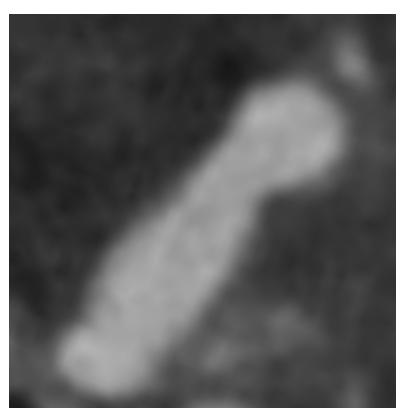

(a) Original data

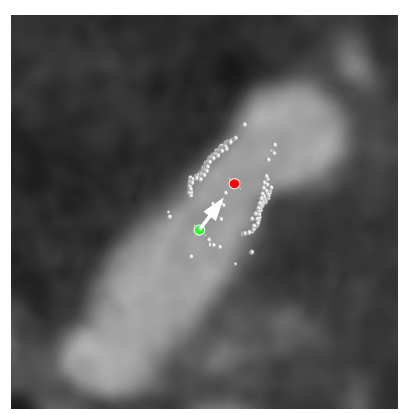

(b) Candidate points

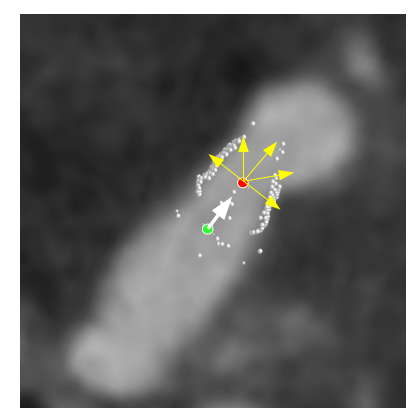

(c) Test cylinder axes

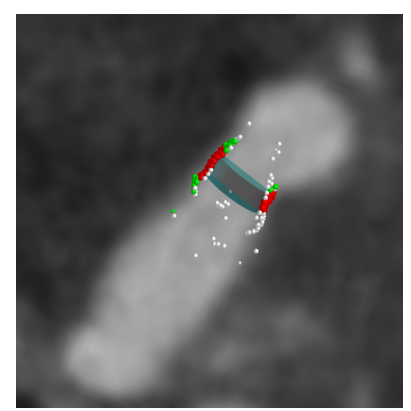

(d) Final cylinder and points

Figure 1: Outline of RBT vessel tracking algorithm. (a) Cut plane through the original 3DRA data; (b) Parameter update: Starting from $C_{0}$ (green dot) and $\overrightarrow{d_{0}}$ (white arrow), the new center $C$ (red dot) is found. $\vec{d}=\overrightarrow{d_{0}}$. Candidate points (white dots) are extracted at the vessel surface by casting rays in the volume, from $C$. Only the points around the displayed cut plane are shown. (c) $N_{d}$ directions are tested for the cylinder axes (yellow arrows). One fitting cylinder is found using RANSAC, per axis. (d) Best cylinder found, together with its consensus set (green dots) and the final points used to set the cylinder height.

\subsubsection{RANSAC-Based Cylinder Estimation}

The elementary tracking step consists in fitting a cylinder to the local vessel shape. We first suppose that this step was carried out and an initial cylinder of center $C_{0}$, axis direction $\vec{d}_{0}$, radius $r_{0}$ and height $h_{0}$ was fitted. Hereafter, we describe how the next cylinder is found.

The center $C$ and the direction $\vec{d}$ of the cylinder are first predicted: $C=C_{0}+\left(h_{0} / 4\right) \vec{d}_{0}$, and $\vec{d}=\overrightarrow{d_{0}}$. The radius $r$ is bounded to be in $\left[r_{0} / 2,3 r_{0} / 2\right]$. An initial set of points are extracted using ray casting: $N_{r}$ rays, with a length of $4 r_{0}$, equidistributed on a sphere, are casted from $C$; then, the point of minimum of the directional gradient is computed along each ray*. This extraction scheme is very similar to taking the points of minimal gradient along the columns of a Bounded Spherical Projection (BSP) image ${ }^{2}$ (see Figure 1b).

Then, a cylinder is fitted to the points, using RANSAC. ${ }^{5}$ Chaperon et al. ${ }^{5}$ estimate the cylinder axis direction from the normals at the input points. This may fail at curved sections of vessels for example. Also, in our case, normals would be estimated as the image gradient at the extracted points. Such an input can be noisy at some places, making it hard to get a trustful cylinder direction. We therefore followed a strategy similar to Friman et al. ${ }^{4}$ for robustness reasons (see Fig. 1c): $N_{d}$ directions are tested, equidistributed on the half-unit sphere oriented along $\vec{d}$. Each direction is associated by RANSAC to a cylinder respecting the constraint on $r$, using a direct formula giving the cylinder center and radius knowing its direction. ${ }^{6}$

Pruning the candidate cylinder directions drastically speeds up the process. The $N_{d}$ directions are reviewed by increasing angle with $\vec{d}_{0}$. As soon as a cylinder presents with an inlier rate above $p_{i n l}$, the algorithm stops and returns the cylinder. In case of failure, the algorithm returns the cylinder with the maximum inlier rate if it is at least $p_{i n l} / 2$ or otherwise, the algorithm returns that no valid cylinder could be found. Finally, the center $C$ of the returned cylinder is refined as the median point of its consensus set, and its parameters (center, radius and direction) are refined with Powell's optimization algorithm, to get a smoother sequence of cylinder directions. A new set of points is extracted by ray casting from $C$ and the subset $\mathcal{P}$ of the inlier points is returned with the cylinder. Its height is set so as to encompass $75 \%$ of $\mathcal{P}$ (see Fig. 1d).

\subsubsection{Tracking}

The tracking is initialized manually by a point $C_{0}$. Most vessels irrigating the brain are vertical: $\vec{d}_{0}$ is the upward direction. A rough estimation of the radius $r_{0}$ is also supplied by the user as well as an inlier rate $p_{\text {inl }}$. The

*Blood vessels are bright on a dark background in 3DRA 
tracking is stopped when no valid cylinder can be found or when it turns back. The other branches are added in a similar way. A point is placed manually on the new vessel, close to the bifurcation. The vector linking this point to the nearest point on the parent vessel centerline provides the direction for the new vessel. The vessel tree is built up by assembling the tracked branches. As a result, only one point per vessel is necessary, hence according to Schaap et al. ${ }^{7}$ classification, our method belongs to the category of algorithms with minimal user-interaction (category 2). For comparison, the MHT algorithm is assigned to category 3: interactive extraction, since a seed point, an initial tracking direction and an estimate of the vessel radius are required.

The algorithm output is thereafter threefold: the centerline is provided by the centers of the successive cylinders, each cylinder gives a robust estimation of the local vessel radius, and a dense sampling of the vessel surface is provided by the cylinder inliers.

\subsection{Validation: Material and methods}

\subsubsection{Image Data}

A set of 10 patient data was used for validation. Each patient data set consisted of a 3DRA acquired on a vascular C-arm (Innova 4100, GE Healthcare) during the intra-arterial injection of the internal carotid artery. Each 3DRA volume presents as a $512^{3}$ isotropic voxel cube, between $0.18-0.22 \mathrm{~mm}$ voxel size. A total of 744 vessels and between 55-96 vessels per patient were examined.

The complex morphology of the vasculature makes any comparison of segmentation outcomes difficult. In the case of multibranch tracking algorithms, it is necessary to set branches into correspondence at each bifurcation, and to handle possible missing branches, which is difficult to automate. In our case of single branch tracking algorithms, the algrithms to compare may follow different branches at bifurcations, which is very hard to distinguish from tracking errors. As a result, only comparisons of vessel sections in-between bifurcations. For the sake of comparison, the algorithms were both initialized with the same four points, placed manually on each vessel section: one seed point $C_{0}$ was placed at the proximal end of the vessel; the vessel direction $\vec{d}_{0}$ was estimated by placing a second point around the vessel axis, a little distal from $C_{0}$; and the vessel radius $r_{0}$ was estimated as the distance between two diametrically opposed points placed on the vessel surface, in a cut plane showing the vessel cross-section around $C_{0}$.

The criterion to include a vessel in the validation set was that it could be tracked visually through successive cut planes in the 3DRA volume data. Only the internal carotid artery (the main vessel) was excluded because its radius is too large for the MHT algorithm used as the reference algorithm (see below). Note that our tracking algorithm gives visually excellent result on this large artery with the same parameters.

\subsubsection{Parametrization of our algorithm}

The RBT procedure was run for each vessel with the following parameters: the inlier rate threshold $p_{i n l}$ was $70 \%$, a relative threshold equal to $10 \%$ the local cylinder radius was taken on the distance to the fitted cylinder for the outlier rejection test; the number of rays thrown to extract points was $N_{r}=162$, and the number of axis directions tested was $N_{d}=81$. A minimum of 220 tests and a maximum of 500 tests were set for RANSAC. Note that the same parameters were used for all vessels, let they be rather large or small.

Though our segmentation algorithm only needs one point per vessel, $C_{0}, \vec{d}_{0}$ and $r_{0}$, as estimated above, were used to initialized our tracking procedure in order to be in the same conditions as the reference method.

\subsubsection{Reference method}

For comparison, the vessels were segmented with the Multiple Hypothesis Tracking (MHT) Algorithm, ${ }^{4}$ available on MevisLab software ${ }^{\dagger}$ under the TubularTracking module. The manual seed $C_{0}$ and direction $\overrightarrow{d_{0}}$ were used to track each branch. The single hypothesis tracking option was set (one single vessel to track). The tracking step was set to 0.1 the radius of current tracking radius in the MHT, the maximum search angle angle between 2 tracking steps to $85^{\circ}$ and the number of search angles was 5. A maximum of 2000 iterations were allowed and all other parameters initialized to default values.

${ }^{\dagger}$ http://www.mevislab.de 
The MHT algorithm proved to be quite sensitive to the allowable bounds set onto the radius. To have MHT working on the most possible vessels, the radius was allowed to vary between 0.25 to 1.25 times the estimated radius $r_{0}$ at the seed point.

Besides, since the reference MHT method has no efficient stopping criteria, the resulting centerline was cut manually where the vessel visual tracking was not reliable anymore.

\subsubsection{Validation protocol}

Since we noticed a certain amount of tracking failures for MHT, we first visually assessed the tracking success. A tracking was considered as successful if the resulting centerline remained inside the vessel of interest. Otherwise, the result was considered as a failure. This first evaluation resulted in a sucess rate in percents (see Table 1).

However, the first examination was tough for MHT. In some cases, rated as failures, the MHT successfully tracked the proximal section of the vessel, but leakage was commonly observed where neighboring dense structures misled the tracking. Since the successfully tracked portion was sometimes quite long, a second experiment was set up. Note that no leakage was noticed for RBT on the entire data set.

In this second experiment, these MHT tracking results, considered as failures in a first place, were visually cut before the problem occurred. According to the length of the detected centerline, results, both for MHT and RBT, were classified as: short, medium, long if the centerline was respectively correct along 1-33\%, 33-66\%, more than $66 \%$ of the visually estimated length of the targeted vessel. A second evaluation was therefore performed, recomputing the success rate, while only taking into account the medium and long trackings. Moreover, successful tracking lengths were computed and compared (see Table 2).

A third evaluation was carried out, focusing on a quantitative assessment of the centerline detection accuracy. The Average Symmetric Surface Distance (ASSD) ${ }^{8}$ was used. Only vessels labelled as medium or long were considered, since we aimed at reporting figures on significant trackings. The ASSD measure translates the mean error between two centerlines. Its computation required to find the centerline sections that were potentially common to both. Each point on the centerline of the ground truth (MHT) was selected as a measure point if the test centerline (RBT) came enough in its vicinity. Each measure point $\left\{P_{i}\right\}_{i=1, \ldots, N}$ was associated with its closest point $\left\{Q_{i}\right\}_{i=1, \ldots, N}$ on the test centerline. Symmetrically, measure points $\left\{Q_{j}^{\prime}\right\}_{j=1, \ldots, M}$ were selected on the test centerline, and their corresponding point $\left\{P_{j}^{\prime}\right\}_{j=1, \ldots, M}$ were computed on the ground truth. The ASSD was thereafter computed as follows:

$$
A S S D=\frac{1}{N+M}\left\{\sum_{i} d\left(P_{i}, Q_{i}\right)+\sum_{j} d\left(P_{j}^{\prime}, Q_{j}^{\prime}\right)\right\}
$$

where $d(P, Q)$ is the Euclidean distance between points $P$ and $Q$.

Two ASSD measures were carried out with different distance thresholds: first, a fixed value of 3 voxels and second, the mean radius of the targeted vessel was taken as an adaptive threshold. Indeed, the former is suitable for large vessels and the latter is a more appropriate choice to consider the scale of vessels and avoid taking into account erroneous centerlines at leaking portions on small vessels. Moreover comparing both measures enables to assess a potential influence of the distance threshold on the result. For the latter measure, the estimation of the mean radius was given by our algorithm, since it appeared more trustful than MHT's estimation (see Fig. 4).

\section{RESULTS AND DISCUSSIONS}

Table 1 reports on the first experiment. A total number of 744 vessel sections were tracked on the 10 patient data included in our experiments. For the visual assessment, the overall performance of RBT algorithm was $94 \%$, confirming its efficiency and strength. In constrast, the MHT algorithm reached a success rate of $65 \%$. This rather poor performance for MHT is the resultant of a lack of stopping criterion, which made MHT keep on tracking, even wrong vessels, when the target vessel became hardly visible. Leakages, due to neighboring structures, were the cause of many failures. On the contrary, the RBT procedure dealt well with this kissing vessel problem. Fig. 2 illustrates the difficulty for MHT to sometimes handle the kissing vessel problem and the robustness of our method to such topological difficulties. 


\begin{tabular}{|c|c|c|c|c|c|c|c|c|c|c|c|}
\hline Patient & 1 & 2 & 3 & 4 & 5 & 6 & 7 & 8 & 9 & 10 & Average \\
\hline Number of vessels & 59 & 57 & 75 & 69 & 82 & 80 & 55 & 95 & 76 & 96 & 74.4 \\
\hline MHT \% & 67 & 72 & 66 & 74 & 60 & 59 & 48 & 67 & 74 & 62 & 65 \\
\hline RBT \% & 97 & 90 & 99 & 81 & 100 & 96 & 85 & 92 & 96 & 99 & 94 \\
\hline
\end{tabular}

Table 1: Success rate of MHT and RBT on a data set of 10 patients. A total of 744 test vessels were used to evaluate both algorithms. The average success rate for MHT and for our algorithm is respectively $65 \%$ and $94 \%$.

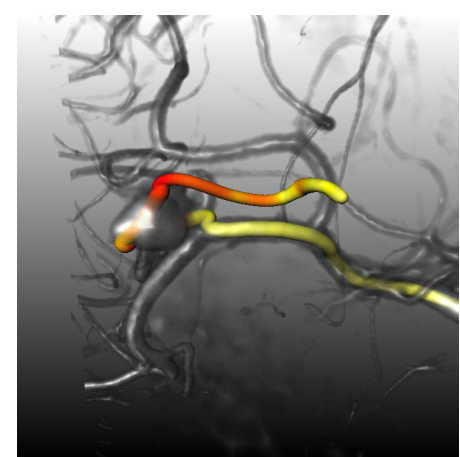

(a) MHT result

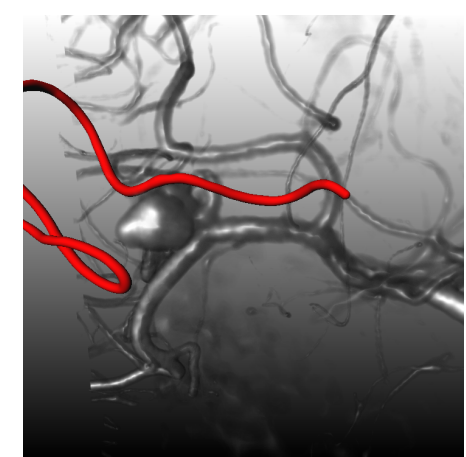

(b) RBT result

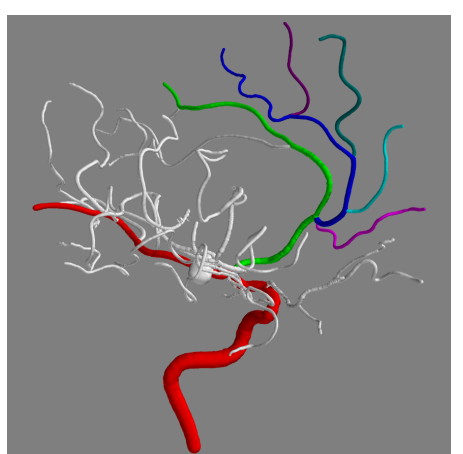

(c) Full result (32 arteries)

Figure 2: Comparison of MHT (left) and our algorithm (middle) with regard to a kissing vessel case on Patient 1: a blood vessel runs along the aneurism sack. Our RBT algorithm successfully handles this case, while MHT gets lost in the aneurism. (right) 32 arteries segmented on this patient data with our algorithm.

The second evaluation took into account the actual tracked length, to assess the success rate. In particular, short trackings that were counted as successes in the first evaluation, were here discarded. Table 2 summarizes its results. As expected, MHT increased its performance up to $69 \%$, since some trackings were previously rated as failures due to leakage issues, though they were correct on the proximal vessel section. On the contrary, RBT performance slightly dropped to $89 \%$. This abatement could be mostly explained by RBT stopping prematurely due to drops in image intensity, as illustrated in Fig. 3.

When both algorithms succeeded, on average, RBT went further $(725.5 \mathrm{~mm})$ than MHT $(688.6 \mathrm{~mm})$. Nonetheless, MHT definitevely delivered a lesser number of successful trackings which impacted negatively the average tracked length per patient up to $778.6 \mathrm{~mm}$ against $1446.1 \mathrm{~mm}$ for RBT. Table 2 also reports quantitative ASSD measures. The average ASSD between both centerlines, with both 3 voxels and the mean radius of the targeted vessel, was below one voxel. This showed that the proposed method produced similar results as the MHT.

On one hand, our RBT algorithm showed the same behaviour over all cases. The most important observation is that it handled nicely the kissing vessel problem. Vessels of varied diameters could also be tracked with the same set of parameters. Nevertheless, our proposal reached its limits when struggling with drops in image intensity. On the other hand, the MHT algorithm coped especially well with gaps but it was very sensitive to both bad initialization and radius variation along blood vessels. A propos this limitation, the user-defined radius estimation bound could lead to a saturation of the radius estimation and ineluctably to a wrong estimation of the centerline as pointed out in Fig. 4. In contrast, RBT used the last estimated radius to restrain the estimate. Thereby, a good behaviour was noticed when contending with variations of density along vessels while the MHT centerline estimation was heavily disturbed (see Fig.5).

One of the reasons we identified as potentially impeding MHT performances is the fixed tracking step that it uses. The increase in resolution in 3DRA implies that we try to track very small vessels $(0.5 \mathrm{~mm}$ in diameter or less). Such vessels present with a very high tortuosity and potentially acute bends. RBT defines the tracking step as the quarter of the height of the last fitted cylinder. But since the height is adapted to the longitudinal extension of the inlier point set, fitted cylinders are shorter in twisted and bent portions of the blood vessels, thus naturally adapting the tracking step. 


\begin{tabular}{|c|c|c|c|c|c|c|c|c|c|c|}
\hline Patient & 1 & 2 & 3 & 4 & 5 & 6 & 7 & 8 & 9 & 10 \\
\hline \multicolumn{11}{|l|}{ MHT } \\
\hline $\mathrm{M} \& \mathrm{~L}(\%)$ & 75 & 71 & 73 & 75 & 65 & 65 & 57 & 67 & 77 & 63 \\
\hline TL (mm) & 765.2 & 601.5 & 758.7 & 726.5 & 818.7 & 1086.9 & 555.4 & 906.1 & 688.1 & 879.0 \\
\hline CL (mm) & 697.1 & 523.3 & 697.5 & 512.4 & 810.2 & 1074.1 & 389.0 & 834.1 & 581.8 & 766.3 \\
\hline \multicolumn{11}{|l|}{ RBT } \\
\hline $\mathrm{M} \& \mathrm{~L}(\%)$ & 94 & 79 & 97 & 77 & 99 & 95 & 82 & 84 & 89 & 91 \\
\hline TL (mm) & 1120.4 & 1063.6 & 1383.3 & 1097.6 & 1752.7 & 2373.6 & 833.9 & 2121.3 & 1420.6 & 1294.0 \\
\hline $\mathrm{CL}(\mathrm{mm})$ & 642.6 & 540.5 & 731.2 & 515.9 & 915.2 & 1164.7 & 403.6 & 914.2 & 644.7 & 782.6 \\
\hline \multicolumn{11}{|l|}{ ASSD } \\
\hline$\mu_{<3}(\mathrm{~mm})$ & 0.20 & 0.21 & 0.16 & 0.17 & 0.16 & 0.16 & 0.18 & 0.19 & 0.16 & 0.20 \\
\hline$\mu_{<\bar{R}}(\mathrm{~mm})$ & 0.20 & 0.20 & 0.16 & 0.17 & 0.16 & 0.16 & 0.19 & 0.19 & 0.16 & 0.20 \\
\hline
\end{tabular}

Table 2: Success rate of MHT and RBT algorithms with tracking results classified as medium and long (M \& L). The mean success rates for MHT and for our RBT algorithm are respectively $69 \%$ and $89 \%$. Furthermore, the mean ASSD, with a distance threshold of 3 voxels $\left(\mu_{<3}\right)$ and the mean radius of the targeted vessel $\left(\mu_{<}\right)$, between the extracted centerlines by both methods is below one voxel (0.17-0.18 mm). The comparison length (CL) corresponds to the total length of centerline used to compute ASSD on vessels successfully tracked by both methods. On average the total tracked lengths (TL) for MHT and RBT are respectively $778.6 \mathrm{~mm}$ and 1446.1 mm.
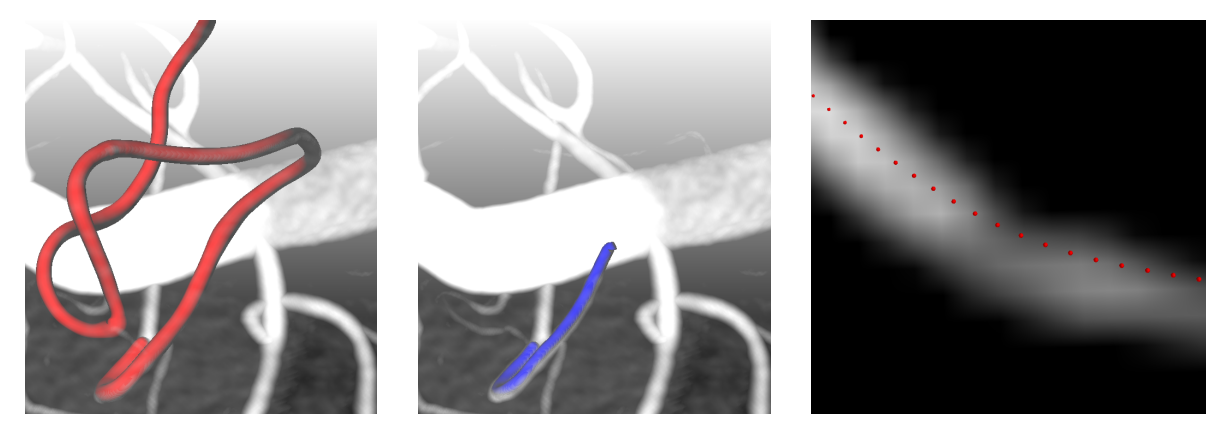

Figure 3: MHT deals well with drops in image intensity. (left) MHT result; (middle) RBT result: the tracking stops too early. (right) a cut plane along the MHT centerline shows the drop in image intensity in the vessel. The tracking was performed on a branch of the Middle Cerebral Artery of Patient 10.

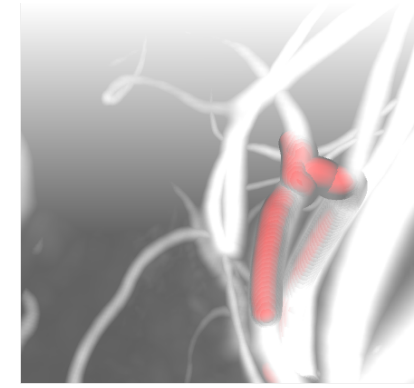

(a) MHT result with radius saturation

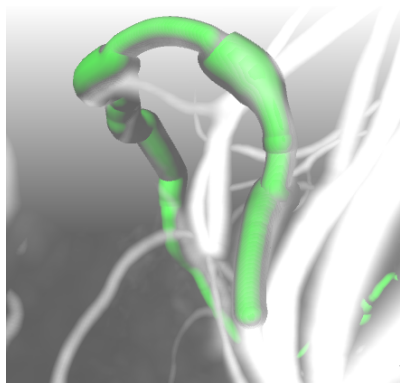

(b) MHT result without radius saturation

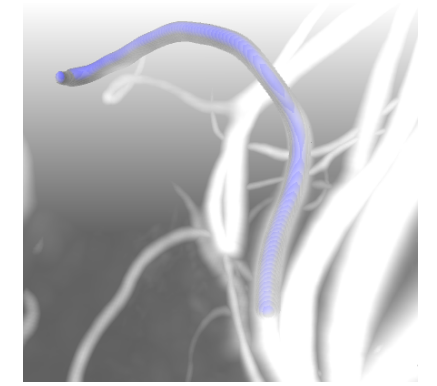

(c) RBT result

Figure 4: Influence of user-defined radius estimation bound on MHT tracking where underestimation of the vessel radius leads to an innacurate outcome on Patient 5: (left) the upper bound is reached leading to leakage issues and (middle) computation with larger values avoid this problem. (right) Our RBT algorithm successfully tracks the vessel without resorting to this information. 


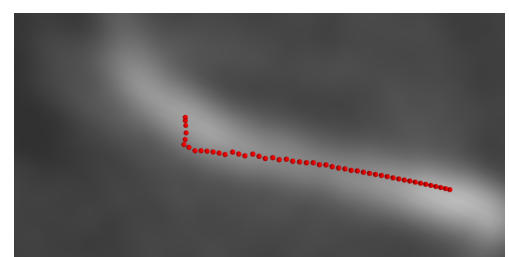

(a) Cut plane along the centerline of Fig.4a

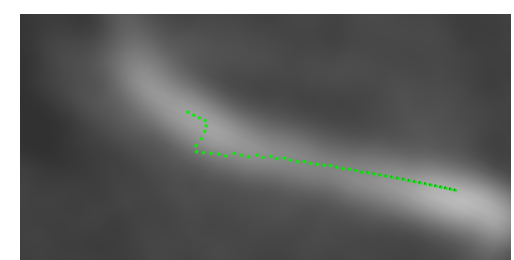

(b) Cut plane along the centerline of Fig. $4 \mathrm{~b}$

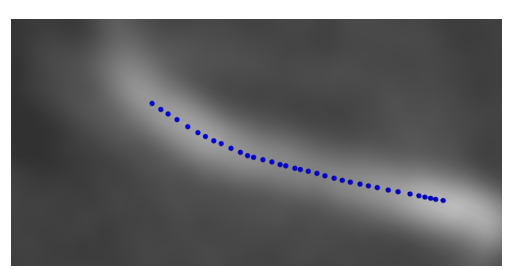

(c) Cut plane along the centerline of Fig. $4 \mathrm{c}$

Figure 5: Plane views of Fig.4: the detection of the centerline for the MHT on a fuzzy portion of $0.8 \mathrm{~mm}$ of diameter, with saturation of the radius (left) and without saturation (middle), is disturbed whereas our algorithm detects correctly the centerline.

\section{CONCLUSION}

The general context of our work is interventional neuroradiology. While most previous works present segmentation results on CTA or MRA data, we only addressed the segmentation of 3D Rotational Angiography (3DRA) volume. Indeed, 3DRA is the modality of choice for interventional radiologists that routinely acquire 3DRA data before, during and after the treatment. While 3DRA is arguably of a much higher quality than CTA and MRA, which would supposedly ease the segmentation task, noise and artefacts are still present. Moreover, even in 3DRA, and as in MRA and CTA, our aim remains the segmentation of vessels whose radius is about the voxel size. A state of the art MHT vessel segmentation algorithm ${ }^{4}$ was used as a reference for validation. The performance of MHT were below our expectations, when applied to 3DRA, which supports our claim that 3DRA presents with specific issues regarding blood vessel segmentation: improved blood vessel visibility implies more kissing vessels, and vessels with a much stronger tortuosity.

The proposed algorithm for blood vessel segmentation, called RBT, builds a vessel tree structure, without imposing the vessels to be of circular cross-section. Furthermore, robustness is incorporated in the overall tracking procedure by using RANSAC to handle outliers in candidate points at the vessel surface. The results demonstated the strength of the proposed method as well as its ability to accurately detect centerlines. In particular, RBT proved to be able to capture very complex vascular topologies and to be very robust to the kissing vessel issue. In addition, it tracked very tortuous tiny blood vessels. As such, it improves upon previous blood vessel tracking algorithms.

Besides, RBT is also able to continue vessel tracking past bifurcations. After tracking a vessel, a child vessel is segmented by providing a seed point on the parent vessel. This point is connected to its closest point on the centerline of the parent vessel, which provide a initial direction for the tracking. The initial radius is the same as that of the parent vessel at the connecting point. This rough initialization is possible due to RBT being very robust. It did not work with MHT, that required tighter initial parameter values. Furthermore, RBT was even robust enough for us to use the same set of parameters (aside from center, direction and radius) for all patients and vessel. These characteristics of our method minimize user interaction and allow for segmentation of vessels of interest to the user. This counterbalances the fact that RBT does not automatically handles bifurcations. We would even argue that it is a clinical advantage since the radiologist is often interested in only a part of the vasculature (the one related to the pathology), that he/she would probably like to choose and control. Anyway, user interaction would be necessary, even with a so-called fully automated procedure, to correct for potential errors. Finally, the proposed segmentation algorithm seems to be easily extended to other modalities. Preliminary outcomes showed a promising behaviour of our algorithm on CTA data.

A path to improve RBT relies on a recognized strength of MHT. MHT allows the tracking to locally degrade the fitting score, as long as a raise occurs. As a result, MHT resists to local drops in the density along a vessel. However, this strategy prevents MHT for stopping per se as it too greedly follows hints of vesselness on the data. Our method stops as soon as no cylinder is found with the minimum percentage of inliers and may occasionnaly stops prematurely. In order to increase the robustness of our proposal, we need to integrate an exploration phase 
during the tracking procedure. A difficult compromise would have to be found between our stopping criteria and a greedy MHT exploration.

A final word should be said on the segmentation characteristic of RBT. It is a tracking algorithm since the vessel centerlines are extracted, building a vessel tree with little manual interaction. But, as MHT, the local vessel radius is also estimated. However, RBT goes further since it also provides a set of points that reliably lie on the local vessel surface. These points provide a rather dense sampling of the vessel surface. Our current and future work aim at providing a complete vessel implicit surface representation that interpolate these points.

\section{Acknowlegments}

The authors would like to express their gratitude to Pr. René Anxionnat who provided the data for evaluation. Figures 2a, 2b, 3 (left), 3 (middle), and 4, were produced with MeVisLab software (http://www.mevislab.de). Figures 2c, 3 (right), and 5 were generated with Mayavi software (http://mayavi.sourceforge.net).

\section{REFERENCES}

[1] Lesage, D., Angelini, E., Bloch, I., and Funka-Lea, G., "A review of 3D vessel lumen segmentation techniques: Models, features and extraction schemes," MedIA 13(6), 819-845 (2009).

[2] Wong, W. and Chung, A., "Probabilistic vessel axis tracing and its application to vessel segmentation with stream surfaces and minimum cost paths," MedIA 11, 567-587 (2007).

[3] Li, H., Yezzi, A., and Cohen, L., "3D multi-branch tubular surface and centerline extraction with 4D iterative key points," in [MICCAI 2009], LNCS 5761, 1042-1050 (2009).

[4] Friman, O., Hindennach, M., Kühnel, C., and Peitgen, H.-O., "Multiple hypothesis template tracking of small 3D vessel structures," MedIA 14(2), 160 - 171 (2010).

[5] Chaperon, T. and Goulette, F., "Extracting cylinders in full 3D data using a random sampling method and the Gaussian image," in [Vision Modeling and Visualization Conference (VMW'01)], 35 - 42 (2001).

[6] Christian Beder and Wolfgang Förstner, "Direct solutions for computing cylinders from minimal sets of points," in [9th European Conference on Computer Vision (ECCV'06)], LNCS 3954, 135-146 (May 2006).

[7] Schaap, M., Metz, C., van Walsum, T., van der Giessen, A., Weustink, A., Mollet, N., Bauer, C., Bogunovi, H., Castro, C., Deng, X., Dikici, E., O’Donnell, T., Frenay, M., Friman, O., Hoyos, M. H., Kitslaar, P., Krissian, K., Khnel, C., Luengo-Oroz, M. A., Orkisz, M., Smedby, ., Styner, M., Szymczak, A., Tek, H., Wang, C., Warfield, S. K., Zambal, S., Zhang, Y., Krestin, G. P., and Niessen, W., "Standardized evaluation methodology and reference database for evaluating coronary artery centerline extraction algorithms," Medical Image Analysis 13/5, 701-714 (2009).

[8] Schaap, M., Neefjes, L., Metz, C., van der Giessen, A., Weustink, A., Mollet, N., Wentzel, J., van Walsum, T., and Niessen, W., "Coronary lumen segmentation using graph cuts and robust kernel regression," in [Information Processing and Medical Imaging (IPMI'09)], LNCS 5636, 528-539 (2009). 